\title{
Integrated Systems for Commercial and Technological Control of Energy Resources Consumption in Urban Areas and Businesses in Russia
}

\author{
Roman Belousov $^{1}$, Eugene Fiskin ${ }^{1}$, and Margarita Fiskina ${ }^{1}$ \\ ${ }^{1}$ Irkutsk National Research Technical University, Russia
}

\begin{abstract}
The article contains a brief description of the operation principles information and measuring system (IMS) called "KumirResource". It is compatible with different manufactures' equipment, so it can create a network using equipment that is already in place, which is versatile. It can also network with the existing SCADA systems to provide them with necessary information.
\end{abstract}

In today's world, in order to use energy resources more efficiently there is a need to optimize the use of monitoring processes and managing supply chain in real-time. In some instances, there is also a need to add systems of energy resources production to the cycle.

In general, the real-time management is not an issue anymore. There has been a number of successful businesses developing SCADA systems for some time. However, in practice SCADA systems often require using specific and unique equipment, such as detectors, meters, etc. Oftentimes businesses already have similar equipment in place, so its removal, purchasing and installation of the new equipment can be expensive and may take time. There are cases when one business would have several SCADA systems in place, each would have its own task, but all of them would require the same information. Supplying this required information is done by installing several sets of detectors in the same control spot, which results in excessive equipment.

It is obvious that despite effectiveness of the real-time management of modern SCADA systems, there are still issues of information flow, namely:

- SCADA may not be compatible with the existing equipment;

- Different SCADA systems may have issues with proper information flow between each other.

Information and measuring system (IMS) may solve all these issues. It is compatible with different manufactures' equipment, so it can create a network using equipment that is already in place, which is versatile. It can also network with the existing SCADA systems to provide them with necessary information.

For several years, the STC "Kumir" has been developing IMS called IS "KumirResource" that implements the above-mentioned approach.

The following approaches are used in the IMS: 
- Network of batch data transmission and format batches of the measuring devices without transformation is used. Thus, the terminal devices should be a gateway. An abstract query system is used allowing for creating a system regardless of the type of the metering device.

- Data entry/output and system management is done via web interface to create internetoriented metering system.

- BSP system architecture is used to design the system on the whole, which makes the service affordable for businesses in Russia; this will facilitate introduction of internet-based solutions to the housing and utilities companies.

- Communication with the metering stations is done using GPRS technology.

- Access to multiple devices. This allows us to query a large number of energy metering stations at a time.

- Data collected form the metering stations is stored and analyzed on a server, which makes it possible to store and analyze a big volume of information. The operator can easily retrieve the required data.

- Own web server is available to report to all interested parties via Internet.

- Data backup. In case the server fails, all data is preserved, which is hard to implement if every user keeps their own information locally.

- IMS "Kumir-Resource" is built on client server technology; client applications are web browsers, and web server is the system's interface.

- Cross-platform implementation: IMS "Kumir-Resource" is based on a Software-as-aService (SaaS) model. SaaS model means that software is not purchased, but it is rather part of service. The benefits of SaaS include:

- no system administration expenses;

- no setup required

- no support and renewal required

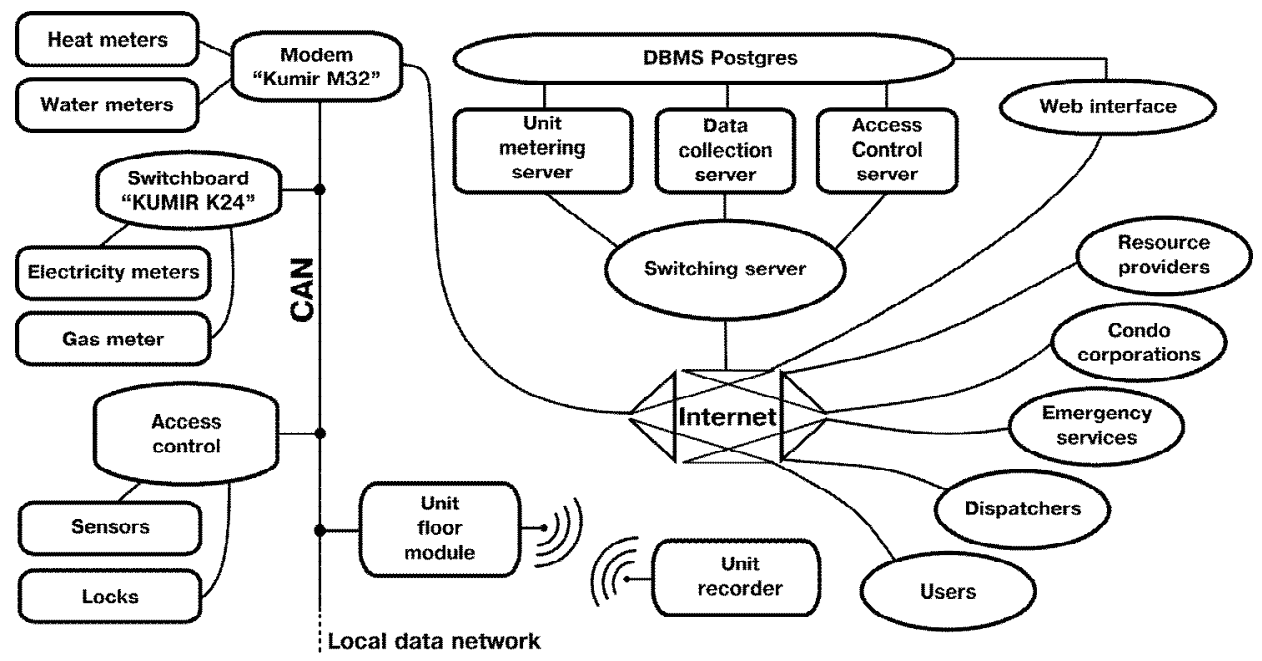

Fig. 1. Illustrates the architecture of the IMS "Kumir-Resource". 


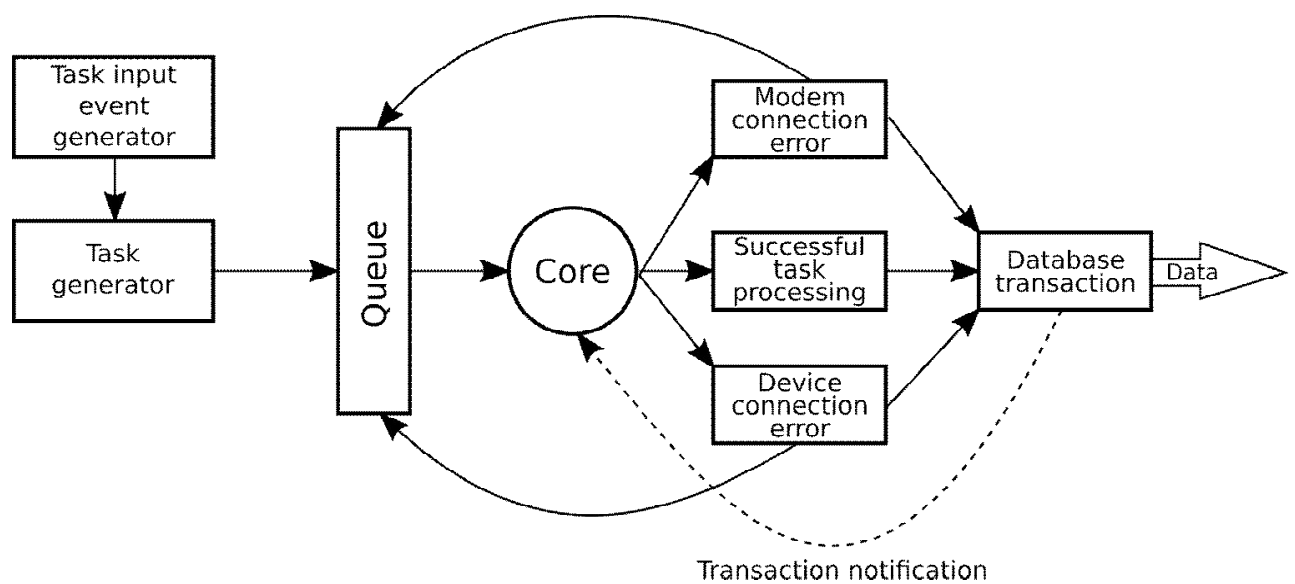

Fig. 2. Illustrates the functional scheme of the IMS.

In order to determine the efficiency of the system, an imitation model was built. One of the most effective and widely available complex discreet system modelling languages is the GPSS language. It can be successfully utilized in modelling of the systems formalized as systems of queuing. As language objects, the analogues of such standard components of queuing system used as requests, serving devices, queues, etc. Such a set of components facilitates creating complex imitation models while keeping the regular queuing system terminology. This language was selected to build the imitation model.

As seen in Image 2, the model is non-linear and contains feedback branching. The main element of the system is the core. It should be represented at GPSS as a multi-channel device (MCD). This is because the logic of query management of heat meter is the same for any system's source code.

The main task in information and measuring system modelling is to calculate the capacity of the core that manages the querying of the heat meters and data entry in the database.

The results of the modelling are as follows:

- A number of channels in MCD (core) determines the capacity of the system in general and is limited by bandwidth of the main communication channel from mobile carrier to system's server and is 500 channels per $1 \mathrm{Mb}$ of the band.

- The system is immune to communication interference because modelling showed that connection errors even at the rate of $25 \%$ do not decrease the capacity.

The following experiment was made on the dedicated server: simultaneous querying of 1,000 detectors in 100 buildings on 100 streets of 100 cities was modelled. The server load was $18 \%$ thus demonstrating high capacity of the system and possibilities of its scaling.

The abstract architecture of the querying system and the versatile protocol "Kumir-Net" facilitate stable work with detectors, meters, etc. regardless of their internal architecture, i.e, the IMS does not require devices of a specific manufacturer which allows for building IMS on the base of the existing devices.

Along with sharing information with all interested parties by means of web-interface (browser), the IMS can be integrated with SCADA systems to provide them with the data from the terminal metering stations, in the required format as shown in Image 3 . This is achieved by utilizing switching server which does not not only organize work of the IMS itself, but also transforms the identification data from query spots (such as meters, detectors, etc.) into tag sets used by SCADA system. Therefore, the switching server acts like a gateway that provides data exchange between SCADA and IMS. IMS "Kumir- 
Resource" can work with more than one SCADA system simultaneously, which solves the issue of excessive installation of metering devices.

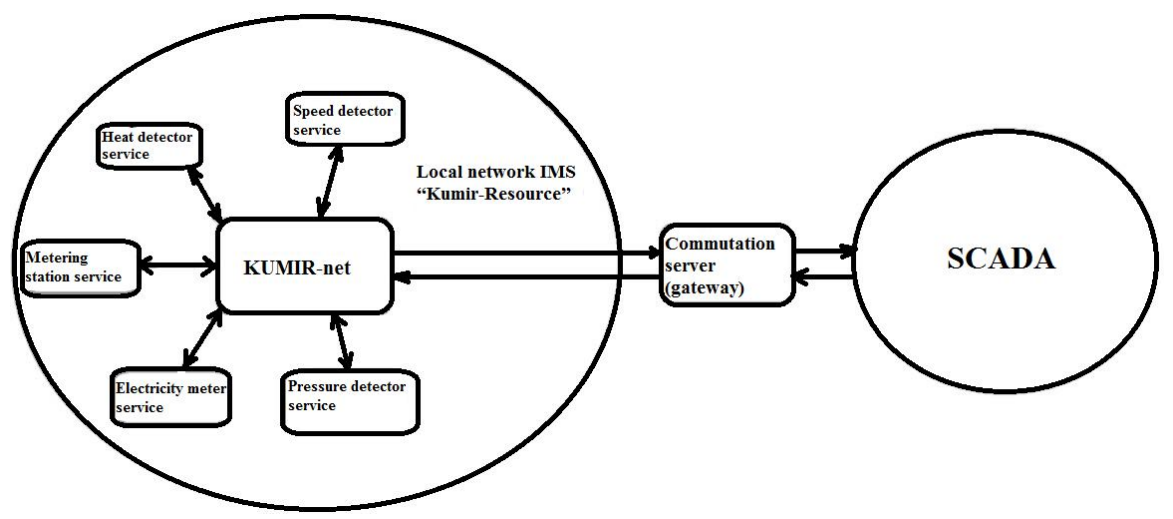

Fig. 3. IMS Integration with SCADA Systems:

\section{References}

1. N.N. Novitsky, M.G. Sukharev, A.D. Tevyashev and others, Pipeline energy systems: Methodical and applied problems of mathematical modeling (Science, Novosibirsk, 2015) [in Russian]

2. R.A. Belousov, I.A. Buzikov, N.N. Klimov, E.M. Fiskin and M.M. Fiskina, Modern technologies. System analysis. Modeling, 3. 19 (2008) [in Russian]

3. R.A. Belousov, A.A. Pinkin, E.M. Fiskin and M.M. Fiskina, EURO-ECO 2015. Das Internationale Symposium "Ökologische, Technologische und Rechtliche Aspekte der Lebensversorgung", 15 (Hannover, 2015)

4. R.A. Belousov, A.A. Pinkin, E.M. Fiskin and M.M. Fiskina, EURO-ECO 2014. Das Internationale Symposium "Ökologische, Technologische und Rechtliche Aspekte der Lebensversorgung", 28 (Hannover, 2014)

5. Jim Pinto. Automation Unplugged: Pinto's Perspectives, Pointers, \& Prognostications (2004)

6. Terrence L. Blevins, Gregory K. McMillan, Willy K. Wojsznis, and Michael W. Brown. Advanced Control Unleashed: Plant Performance Management for Optimum Benefit (2003)

7. J. Rekstad, M. Meir and A. R. Kristoffersen, Energy and Buildings, 35 (2003)

8. B.K . Duncan, B.G. Bailey, Industry Applications, IEEE Transactions, 40 (2004) 